This item was submitted to Loughborough's Research Repository by the author.

Items in Figshare are protected by copyright, with all rights reserved, unless otherwise indicated.

\title{
A simple displacement aptamer assay on resistive pulse sensor for small molecule detection
}

PLEASE CITE THE PUBLISHED VERSION

https://doi.org/10.1016/j.talanta.2020.122068

\section{PUBLISHER}

Elsevier BV

VERSION

AM (Accepted Manuscript)

\section{PUBLISHER STATEMENT}

This paper was accepted for publication in the journal Talanta and the definitive published version is available at https://doi.org/10.1016/j.talanta.2020.122068.

\section{LICENCE}

CC BY-NC-ND 4.0

\section{REPOSITORY RECORD}

Maugi, Rhushabh, Bernadette Gamble, David Bunka, and Mark Platt. 2020. "A Simple Displacement Aptamer Assay on Resistive Pulse Sensor for Small Molecule Detection”. Loughborough University. 
A Simple Displacement Aptamer Assay on Resistive Pulse Sensor for Small Molecule Detection.

Rushabh. Maugi, ${ }^{1}$ Bernadette. Gamble, ${ }^{1}$ David Bunka, ${ }^{2}$ Mark Platt..${ }^{*}$

1. Department of Chemistry, Loughborough University, Loughborough, Leicestershire. LE11 3TU, UK.

*m.platt@lboro.ac.uk

2. Aptamer Group Limited. Bio Centre, Innovation Way, Heslington, York, YO10 5NY, UK. david.bunka@aptamergroup.co.uk 


\begin{abstract}
A universal aptamer-based sensing strategy is proposed using DNA modified nanocarriers and Resistive Pulse Sensing (RPS) for the rapid ( $\leq 20 \mathrm{~min}$ ) and label free detection of small molecules. The surface of a magnetic nanocarrier was first modified with a ssDNA (anchor) which is designed to be partially complimentary in sequence to the ssDNA aptamer. The aptamer and anchor form a stable dsDNA complex on the nanocarriers surface. Upon the addition of the target molecule, a conformational change takes place where the aptamer preferentially binds to the target over the anchor; causing the aptamer to be released into solution. The RPS measures the change in velocity of the nanocarrier as its surface changes from dsDNA to ssDNA, and its velocity is used as a proxy for the concentration of the target. The length of the aptamer and the ability to extract and preconcentrate the nanocarriers using a magnet, is shown to affect the sensitivity. We illustrate the versatility of the assay using the same anchor sequence and Aptamers to the antibiotic Moxifloxacin, and chemotherapeutics Imatinib and Irinotecan. In addition, the proposed assay can be easily extended to detect multiple analytes simultaneously, by utilizing nanocarriers with different diameters. Each sized particle is functionalised with a the same anchor but a unique aptamer. We illustrate this with the simultaneous detection of Imatinib and Moxifloxacin. The strategy could be easily adapted to a range of targets and unlike previous strategies that use aptamer modified nanocarriers, the signal is not dependent upon the tertiary structure of the aptamer-target interaction.
\end{abstract}

Keywords - Aptamer, sensor, resistive pulse sensing, antibiotics, therapeutics 


\section{Introduction}

Aptamers are single stranded nucleic acids, that bind to target analytes through the formation of a sequence specific tertiary structure. ${ }^{1,2}$ They are typically isolated from degenerate chemically synthesised combinatorial oligonucleotide libraries, containing $10^{14}-10^{15}$ different sequences. ${ }^{3-5}$ The process for the isolation of aptamers was first reported in the early 1990's and since the discovery of the in vitro selection process, they have been increasingly advocated as alternatives to antibodies. ${ }^{6}$ They have wide ranging applications; including as therapeutics, bioimaging reagents and as recognition elements in many sensing platforms. ${ }^{7,8}$ Sensing strategies utilise the target induced conformational change in the aptamers tertiary structure, and there has been a move towards "tagless" systems where the binding of the analyte can be monitored without the use of probes.

One emerging technology that is well suited for studying DNA structure is Resistive Pulse Sensing (RPS). The incorporation of aptamer sequences into RPS strategies has expanded its sensing capabilities from DNA sequencing to the detection of small molecules, proteins, pathogens and metal ions. ${ }^{9-11}$ An appealing strategy for utilising aptamers within RPS is to functionalise nanocarriers surfaces with the DNA / peptides. This is particularly appealing when the nanomaterials are superparamagnetic, as the nanocarriers can perform both the purification and sensing roles. Previous work from our group has used the velocity of the carrier as a proxy for the concentration of the target. By adopting a tertiary structure in the presence of the target, the charge density around the nanocarrier is altered. ${ }^{12-14}$ This is measured through a change in translocation velocity and can provide quantitative information. ${ }^{13,15,16}$.

Given that each target and aptamer pair adopt a specific tertiary structure; strategies that rely upon large conformational changes in tertiary structure, may not offer a universal sensing platform. We have shown previously how the change in location and magnitude of the aptamers structure can limit the signal. ${ }^{13,17}$ Here we present an alternative approach which allows any aptamer to be incorporated into the RPS technology; regardless of its affinity and tertiary structure. In this approach, the RPS monitors the presence of ss/dsDNA around the carrier, exploiting the dual nature of the aptamer that switches from Watson-Crick base pairing to specific ssDNA-analyte interactions. The RPS monitors the presence of the target as it induces a conformational change in the aptamer DNA, resulting in displacement of one strand from the nanocarrier's surface. This results in a change from dsDNA to ssDNA. The target induced change in charge density and thus translocation velocity, is independent of the tertiary structure of the aptamer-target complex.

Here we demonstrate the assay for the detection of three small molecule targets; Moxifloxacin, Imatinib and Irinotecan. Moxifloxacin is an antibiotic used to treat a range of infections including respiratory tract infections, cellulitis, endocarditis, meningitis, and tuberculosis. Imatinib and Irinotecan are both anticancer drugs used to treat leukaemia and colon cancer respectively. 
To develop the assay format, we show the effects of changing the aptamer length and packing density on the nanocarriers surface, as well as developing a multiplexed assay with an internal calibration standard, designed to remove lengthy calibrations. The results are a universally applicable, simple and rapid method to quantify small molecules in solution.

\section{Material and methods}

All chemicals are purchased from Sigma-Aldrich, unless stated otherwise. Phosphate Buffered Saline (PBS, P4417) is made up to $137 \mathrm{mM}$. Magnesium chloride (M8266) made with $2 \mathrm{mM}$ and calcium chloride (C5670) at $1 \mathrm{mM}$, here on simply referred to as PBS. Calibration particles (mode diameter $210 \mathrm{~nm}, 1 \times 10^{12}$ particles $/ \mathrm{ml}$ ) and Nanopores (analysis range of $85-500 \mathrm{~nm}$ ) were purchased from Izon Science Ltd (CPC200 and NP200 respectively), the nanopore used in this experiment is NP200 with an analysis range of $85-500 \mathrm{~nm}$. The streptavidin functionalised magnetic nanocarriers (mode diameter $120-150 \mathrm{~nm}$, concentration $1 \times 10^{12}$ particles $/ \mathrm{ml}$ ) were purchased from Ademtech, France (03120). Aptamers against each of the three targets (both full length and minimal functional fragments in each case) and a series of partially complimentary immobilisation oligonucleotides (carrying 5' biotin or amine groups) were all supplied by Aptamer Group Ltd (York, UK). 4-Morpholineethanesulfonic acid, MES hydrate (M5287) made to $50 \mathrm{mM}$ at $\mathrm{pH}$ 6. 1-(3-(Dimethylamino)propyl)-3ethylcarbodiimide hydrochloride (EDC, 22980) purchased from Thermo Fisher. Polystyrene nanocarriers of sizes $150 \mathrm{~nm}, 300 \mathrm{~nm}$ and $350 \mathrm{~nm}$ were purchased from Bangs Laboratories, USA (PC02001).

Aptamer selection.

Selection of the aptamers against the reported targets (Moxifloxacin, Imatinib and Irinotecan) was carried out by Aptamer Group Ltd (York, UK) according to the company's 'Displacement Selection' approach, which was extensively developed and automated, based on the methods previously reported ${ }^{18,19}$ Briefly, iterative cycles of selection and amplification of aptamers targeting the small molecule were conducted starting from a degenerate library of ssDNA oligonucleotides (synthesized by Integrated DNA technologies). In each round, the aptamer library was immobilized on streptavidin coated Dynabeads (Life Technologies) modified with a proprietary immobilization oligo. The target molecule stock $(20 \mu \mathrm{M})$ was prepared in 'Binding Buffer' (10 mM PBS pH 6, containing $137 \mathrm{mM} \mathrm{NaCl}, 2.7 \mathrm{mM} \mathrm{KCl}, 2 \mathrm{mM} \mathrm{MgCl}, 1 \mathrm{mM}$ $\mathrm{CaCl}_{2}$ and $0.01 \%$ Tween 20 ) with $50 \%$ human plasma. This was then incubated with the aptamer-conjugated magnetic beads. Interaction with the target results in a conformational change in the aptamer, causing them to be displaced from the magnetic beads and released into solution. These displaced aptamers are then recovered and amplified by PCR, using the primers (synthesized by Integrated DNA technologies) specific to the $5^{\prime}$ and $3^{\prime}$ ends of the aptamer library. In the first round, 3 nmol of library was used; in subsequent rounds, the enriched fraction of oligonucleotides selected from each round were used as the input ssDNA library. A 
fluorophore was incorporated into the aptamer during PCR by utilizing a $5^{\prime}$ modified primer. This enabled quantification of the amount of aptamer released from the beads during the incubation with the target molecule. The displaced aptamer fluorescence was used as a means of monitoring the refinement of the aptamer library. In total, 1016 cycles of aptamer selection were performed to enrich the population with the target specific aptamers.

The selected aptamer pool obtained from the final selection round was amplified with aptamer specific primers and subsequently cloned into the pJET sequencing vector according to manufacturer's protocols (ThermoFisher Scientific). Chemically competent NEB 5-alpha E. coli were transformed according to manufacturer's protocols (New England BioLabs). After isolating the plasmid DNA, several clones were sequenced by Sanger Sequencing methods by DNASEQ (University of Dundee). Individual Aptamers were identified and assessed using a Displacement assay adapted for Biolayer interferometry on the Octet QK system (ForteBio, Molecular Devices).

Immobilising Aptamers onto nanocarriers

The streptavidin functionalised magnetic nanocarriers are prepared at $1 \times 10^{10}$ particles/ml in PBS as the binding buffer. The aptamer modified nanocarriers are prepared by adding $5 \mu \mathrm{l}$ of the $50 \mu \mathrm{M}$ biotinylated immobilisation oligonucleotide stock and mixing by continuous rotation for 15 minutes, followed by the addition of $5 \mu \mathrm{l}$ of the $50 \mu \mathrm{M}$ aptamer stock and incubation with continuous mixing for a further 15 minutes. The magnetic nanocarriers are magnetically separated, washed and re-suspended with PBS.

Magnetic Separation

After preparation / immobilisation, the aptamer modified nanocarriers are placed on a magnetic rack and incubated for 10 minutes at room temperature, to collect the magnetic particles. The excess aptamer and buffer are removed, and resuspended win the same volume buffer, This process was repeated before the final aptamer modified nanocarriers are re-suspended with fresh buffer.

Addition of Target to Aptamer-loaded Particles

A concentration series of each target molecule was prepared in binding buffer. The concentration range for Moxifloxacin was $0-200 \mu \mathrm{M}$, for Imatinib $0-13 \mu \mathrm{M}$ and $0-7 \mu \mathrm{M}$ for Irinotecan. In the Moxifloxacin assay; Ciprofloxacin was used as a negative control to demonstrate aptamer specificity. For the Imatinib assay; Irinotecan was used as the negative control, and vice versa. These controls were prepared at the highest concentration in the respective dilution series. 100 $\mu$ l of aptamer functionalised nanocarriers at $1 \times 10^{10}$ particles $/ \mathrm{mL}$ were added to each aliquot of target molecule and incubated for 10 minutes. Each assay was carried out in triplicate, and each measurement performed in duplicate capturing a minimum of 200 events per run. 
RPS Analysis

All measurements were carried out using the qNano instrument (Izon Science Ltd, New Zealand) with a NP200 nanopore, using the Control Suite software for data capture. $75 \mu$ l of PBS was added to the lower fluid cell via the side gaps. Care was taken to ensure that no air bubbles were introduced. The upper fluid cell was fixed in place and $40 \mu \mathrm{l}$ of PBS was added. The electric field was applied and left to establish a stable baseline at $115 \mathrm{nA}$. After every measurement, the upper fluid cell was washed out with the electrolyte buffer and a slight pressure was added to the system to remove any residual particles from the samples and prevent any cross-contamination. Due to manufacturing variations in the nanopores, each nanopore was calibrated using a single stretch $(45-47 \mathrm{~mm})$ and voltage of $+0.6 \mathrm{~V}$. The baseline current is noted, and this will be the guideline for taking measurements. All measurements are taken at a single stretch and voltage for comparison purposes with a current of $115 \mathrm{nA}$ used. These values were used to normalise data for all samples run on the same day; using the same nanopore and experimental conditions. The process of normalisation was such that a calibrant or blank was used as a reference, and then the relative speeds to this references are plotted. For the data in figure 2, a calibrant CPC200 particle was used. During aptamer assays the blank particles i.e. the particle with anchor was used as the reference velocity. For figure 5 one of the particles added to the mixture as an internal standard.

Magnetic assisted detection - improving the limits of detection.

Imatinib aptamer modified nanocarriers were placed in $100 \mu$ land $1 \mathrm{~mL}$ of solution containing Imatinib at $5.68 \mu \mathrm{M}$ and $0.568 \mu \mathrm{M}$, respectively.

Multiplexed Assay

Polystyrene nanocarriers of different sizes (150, 300 and 350nm) were prepared at $1 \times 10^{10}$ particles $/ \mathrm{mL}$ in MES buffer at $\mathrm{pH} 6$. Fresh EDC was prepared at $1 \mathrm{mg} / \mathrm{ml}$ in MES buffer and was added to each aliquot of nanocarriers. After 15 minutes $5 \mu l$ of 5 ' amine modified immobilisation oligonucleotide was added to the solution and left to mix at room temperature with continuous mixing (by rotation) for 15 minutes,

A different target specific aptamer was immobilised onto each of the different sized nanocarriers. Aptamer loaded nanocarriers were pelleted by centrifugation $(12,500$ rpm for 10 minutes) and the excess aptamer solution is removed. Fresh buffer is added to the nanocarriers. For the multiplexed assay; the three different sized nanocarriers (each with a different aptamer) were mixed in a vial, in equal proportions.

Specific assay: One vial with the mixed nanocarriers was incubated with the targets by adding $5 \mu \mathrm{l}$ of target molecule at $5 \mathrm{mM}$ was added to an aliquot of mixed nanocarriers and incubated for 10 minutes. Blank: To a second vial of nanocarriers an equivalent volume of buffer was used for comparison. Negative control (Specificity test): To a third vial containing the nanocarriers. linotecan was used as a negative control for assays using the nanocarriers modified with aptamers against Moxifloxacin and 
Imatinib. After mixing at room temperature for 10 minutes, the samples were analysed on the RPS and compared to the internal standard velocity of the $350 \mathrm{~nm}$ sized nanocarrier.

\section{Results and Discussion.}

Within RPS experiments, each translocation of a carrier through the nanopore produces a characteristic pulse shown in Figure 1 . The pulse magnitude $\left(\Delta i_{p}\right)$, is relative to the volume of the nanocarrier, and the width or full width half maximum (FWHM), of the pulse relates to its velocity, Figure $1 \mathrm{~b} .{ }^{20}$ When all parameters i.e. electric field and convection are comparable the velocity of the carrier is proportional to the surface charge or zeta potential of the carrier (assuming electro osmosis remains constant). ${ }^{20,21}$ Here we record the velocity as $1 / T_{0.5}$ values, which we have previously shown capable of monitoring the length and packing density of DNA around magnetic nanocarriers. ${ }^{22}$ The previous work shows how the velocity of the carrier can differentiate ss/dsDNA, ${ }^{20}$ and it is this mechanism that is exploited here. Each of the Aptamers are anchored onto the nanocarrier via the same short anchor ssDNA. Previous Biolayer Interferometry data from Aptamer Group Ltd (data not shown), has shown that in the presence of the target molecule, the aptamer undergoes a conformational change which disrupts the interaction between the aptamer and the immobilised anchor DNA. This disruption leads to the release of the aptamer from the surface. The hypothesis was that the binding of the target to the aptamer would result in aptamer being displacement from the nanocarriers surface shown schematically in Figure $1 \mathrm{c}$, and that the resulting change in surface charge density would produce a measurable difference in nanocarrier translocation velocity through the pore. ${ }^{22}$

a)

b)
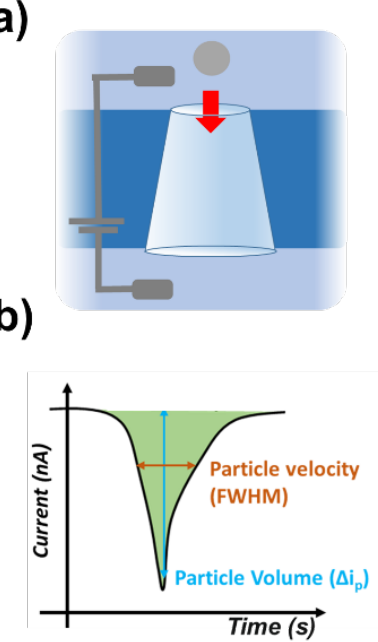

c)

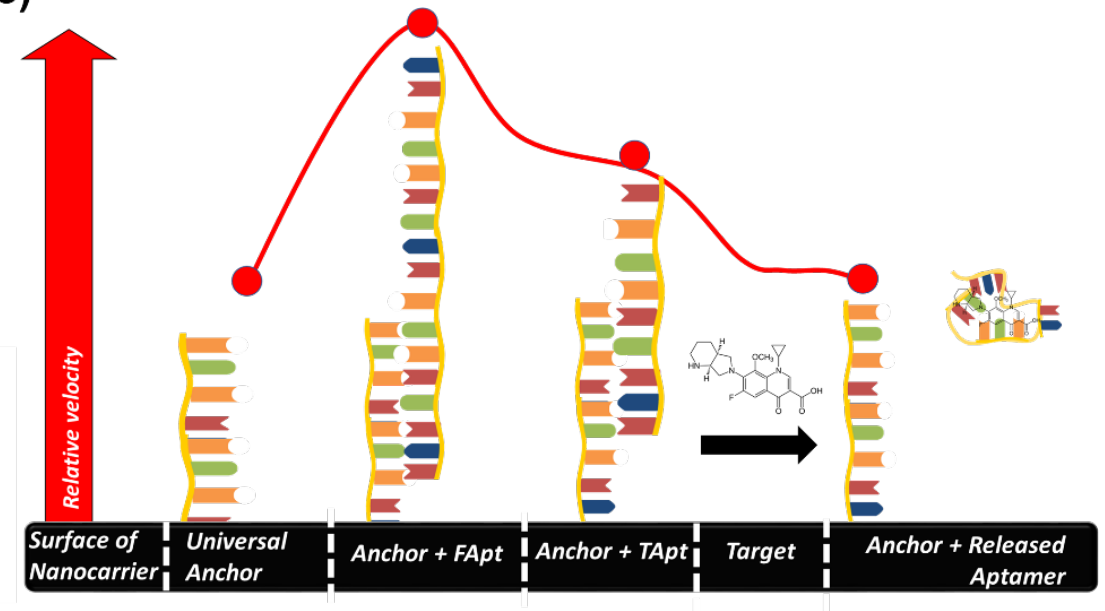

Figure 1:a) Schematic of the translocation of a carrier. b) Example blockade event recorded for each translocation. c) Schematic of the assay procedure. As the target binds to the immobilised aptamer (in a dsDNA complex), the aptamer undergoes a conformational change, disrupting the interaction with the SSDNA anchor. This leaves the ssDNA anchor on the nanocarrier's surface, and results in a decrease in particle velocity.

Each target binding aptamer was provided as both a full-length aptamer (100 bases), and a truncated, minimal functional binding fragment. The length of the truncated 
aptamers were 42 bases 52 bases and 47 bases, for Moxifloxacin, Imatinib and Irinotecan respectively. The anchor sequence was 12 bases long for each assay, and the same anchor sequence was used for all targets.

It has been demonstrated that increasing the density and/ or length of DNA around a carrier can increase the velocity of the particle through the nanopore. ${ }^{20}$ Here the nanocarriers were first modified with the 12 base anchor ssDNA. As the concentration of anchor increases, the velocity of the particles increases, up to circa $200 \mathrm{nM}$, where the velocity reaches a maximum. At this point the surface of the carrier has become saturated with ssDNA. The anchor modified carriers were then incubated with the full length or truncated aptamer sequence (termed FApt, or TApt respectively), to form dsDNA. The relative velocities of these loaded nanocarriers are shown in Figure 2a. As expected the velocity series is FApt $>$ TApt $>$ Anchor $>$ blank. Figure $2 b$ shows an example current trace for the FApt modified nanocarriers. The distribution of the blockade magnitudes are plotted in Figure $2 \mathrm{c}$ for the, anchor, FApt and TApt modified particles. 
a)

b)

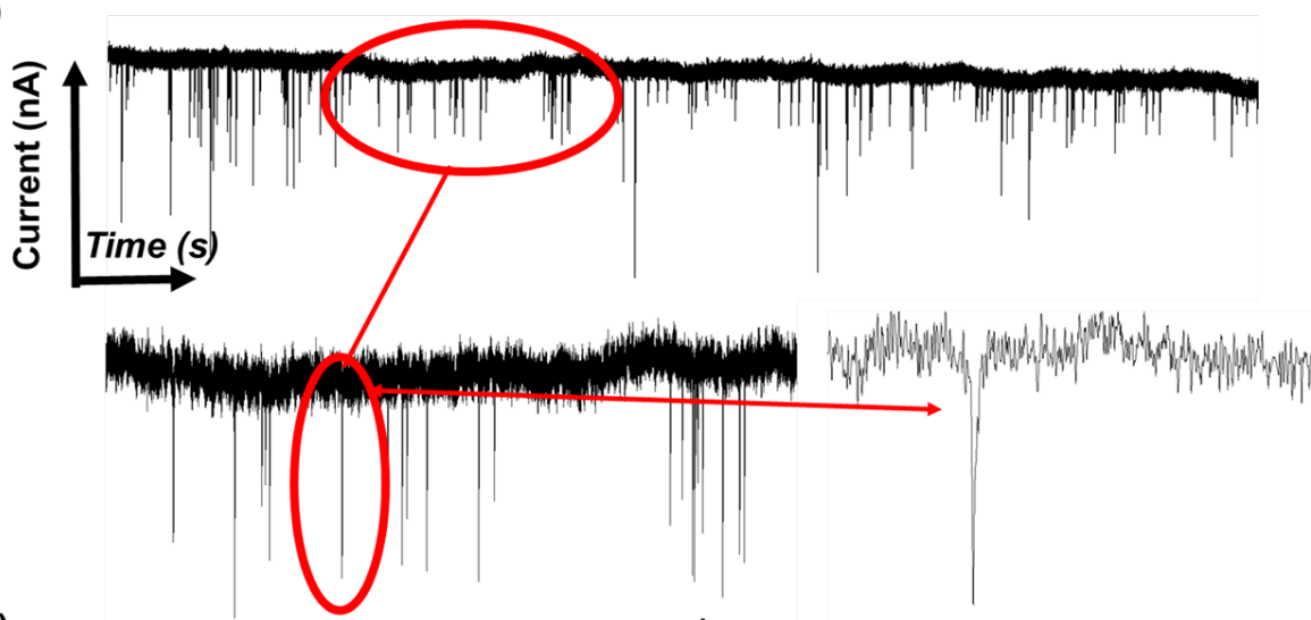

c)
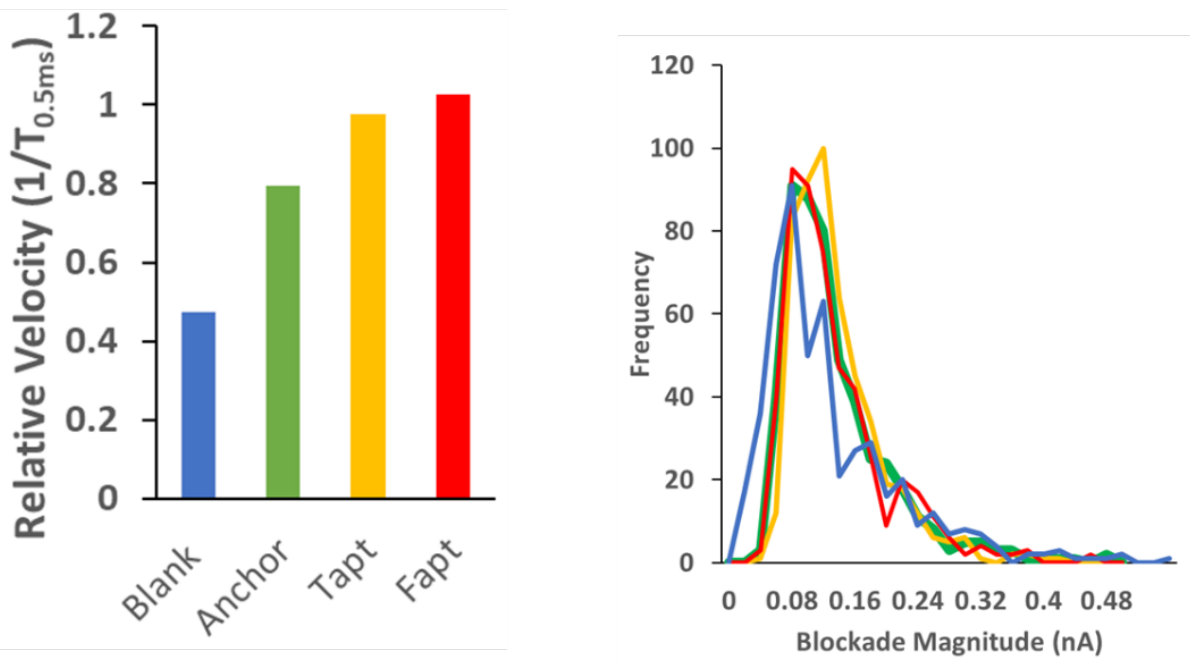

Figure 2: a) Current signal trace for FApt loaded Nanocarriers as they translocate through the nanopore, with an inset of the zoomed image of the trace, scale bar is 2 seconds and $0.5 \mathrm{nA}$. b) Averaged relative translocation velocities of blank nanocarriers (blue) or those modified with the anchor alone (green) and different length aptamers (yellow and red for truncated and full-length aptamer respectively). c) Distributions of the blockade magnitudes of the nanocarriers with different aptamer lengths bound to the nanocarrier, the colour is correlated to figure $2 b$.

The blockade events are above the background current (Figure 2a), and the binding of the aptamers to the carriers does not create a change in particle volume that can be measured under the current setup. The change in velocity was monitored for nanocarriers loaded with FApt(Moxifloxacin) incubated with different concentrations of Moxifloxacin (Figure 3). As the concentration of Moxifloxacin increases, the velocity of the nanocarrier decreases. The velocity continues to decrease up to $200 \mu \mathrm{M}$ Moxifloxacin. Above this concentration, no further reduction in velocity is observed as it had returned to the velocity of the nanocarrier with the anchor alone. We attribute this to the fact that the majority of the aptamer has been removed from the nanocarrier's surface. The values plotted are relative to the nanocarriers loaded with anchor alone, i.e. all velocities have had the anchor velocity subtracted from their value, hence the actual measure average velocity of the particles at $200 \mathrm{mM}$ is not zero. This process allows the truncated and full-length plots to be normalised and overlaid for comparison. Figure 3 also shows the velocity of the FApt(Moxifloxacin) in the 
presence of a non-binding control molecule (Ciprofloxacin open square and triangle plots), to demonstrate that the aptamer is specific and a change in velocity is only seen when the aptamer binds to its target and undergoes the conformational change. The data obtained for TApt(Moxifloxacin) is also presented in Figure 3 (green cicles). This produces the same trend, again the velocity decreases back to that of the carrier loaded with anchor alone.

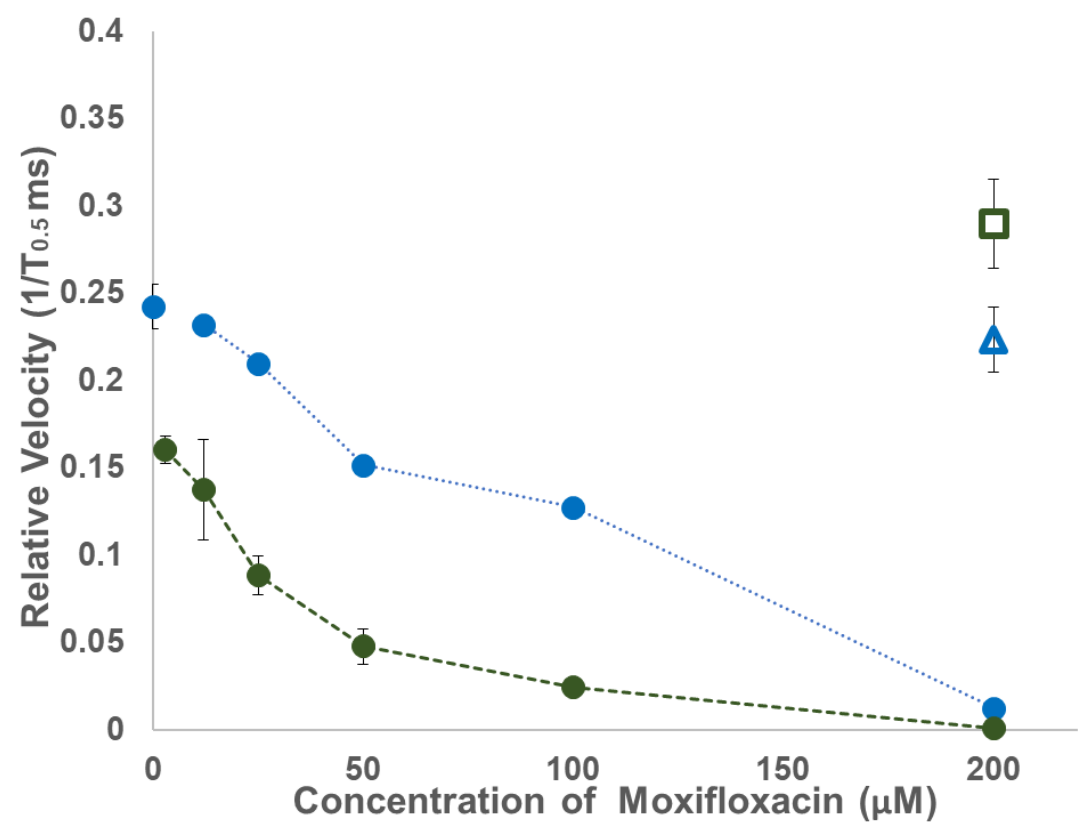

Figure 3: Concentration dependent response curve for the nanocarriers modified with full length aptamer (FApt(Moxifloxacin)) (blue) and the truncated, minimal aptamer fragment (TApt(Moxifloxacin)) (green) when incubated with the antibiotic, Moxifloxacin. The negative control antibiotic target (Ciprofloxacin) was also incubated with the fulllength and minimal fragment aptamer modified nanocarriers (shown as the hollow markers in the respective colours). The error bars represent $1 \times$ STdev of triplicate assays, for Moxifloxacin, $1 \times$ STdev is not large enough to be seen on this plot.

The same principle was then applied using two other aptamers (also developed by Aptamer Group Ltd.) raised against two chemotherapeutic agents (Imatinib and Irinotecan). The change in velocity was recorded at several target concentrations, for both FApt and Tapt, Figure 4. As was seen with the nanocarriers loaded with Moxifloxacin aptamers in Figure 3; as the target concentration increases, the velocity of the carriers through the nanopore, decreases (as the aptamer is displaced from the nanocarrier surface); returning to the velocity of the nanocarriers loaded with anchor alone. 
a)

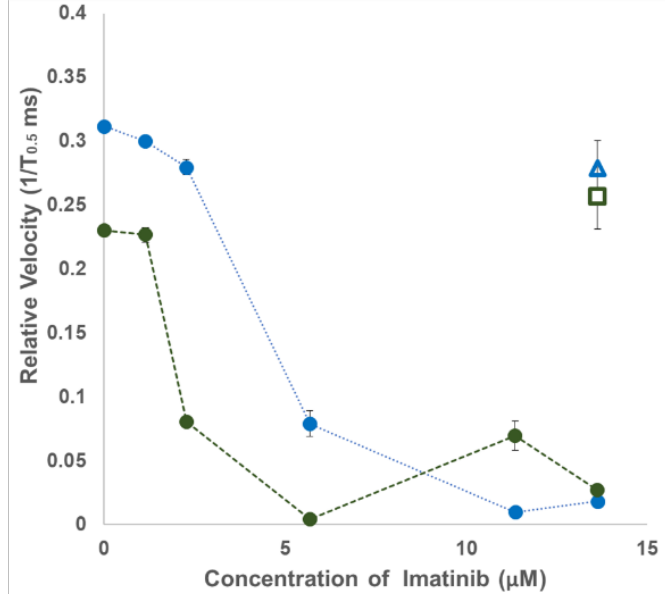

b)

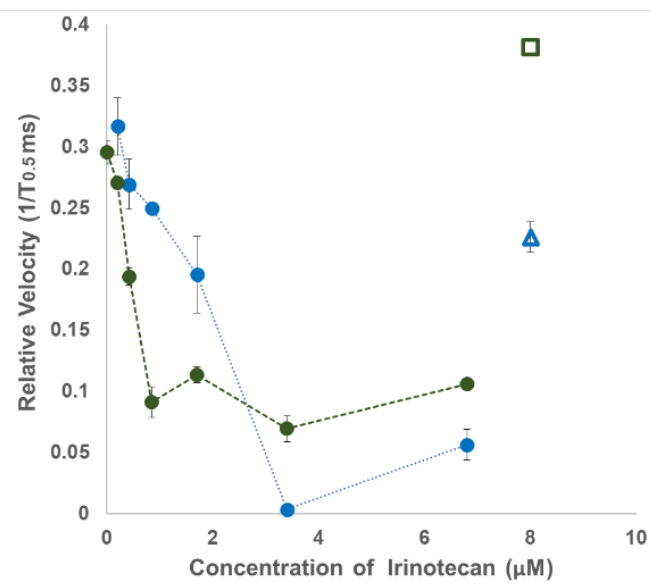

Figure 4: Concentration dependent response curve for the nanocarriers modified with full length aptamer (FApt) (blue) and the truncated, minimal aptamer fragment (Tapt) (green) for the chemotherapeutic drugs (a) Imatinib and (b) Irinotecan. Negative control chemotherapeutic agent (Irinotecan for the anti-Imatinib aptamer and vice versa) was also incubated with the full-length and minimal fragment aptamer modified nanocarriers (shown as the hollow markers in the respective colours). The error bars represent $1 \times$ STdev of triplicate assays, where errors bars are not visible, 1 x STdev is not large enough to be seen on this plot.

To show the effects of using a magnetic nanocarrier to extract and preconcentrate the analyte prior to the detection; an assay was performed as described above using a larger volume containing a lower concentration of analyte. The measured average velocity $\left(1 / \mathrm{T}_{0.5 \mathrm{~ms}}\right)$ of the nanocarriers that had been incubated with $5.68 \mu \mathrm{M}$ and $0.568 \mu \mathrm{M}$ Imatinib was $6.1 \mathrm{~ms}^{-1}$ and $6.2 \mathrm{~ms}^{-1}$ respectively. Whilst larger volumes were needed to measure the change, this illustrates that at least order of magnitude lower target concertation can be achieved. It was also interesting to note that the recorded values are similar which we interpret as a circa $100 \%$ recovery/ detection of the analyte. We acknowledge this was not developed in detail, nor was it the aim for this work. Examples of varying the particle number/ aptamer loading and magnetic separation for RPS can be seen in our previous work. ${ }^{13,15,20,23}$ However it illustrates that a lower LoD can be achieved using different magnetic nanocarrier concentrations and extraction times.

As RPS characterises each nanocarrier through the nanopore, it allows different sized nanocarriers to be added to the same solution, figure $5 a$, where the presence of one nanocarrier will not interfere with the analysis of others. To demonstrate this principle; two different sets of nanocarriers (150 and $300 \mathrm{~nm}$ in diameter) were used. The 150 $\mathrm{nm}$ nanocarriers were functionalised with FApt(Moxifloxacin); while the $300 \mathrm{~nm}$ particles were functionalised with FApt(Imatinib). The functionalised nanocarriers were then mixed, split into separate aliquots and incubated with separately with each of the respective targets. The velocity of translocation was recorded for the multiplexed sample and is shown in Figure 5. The data shows that in the presence of the target molecule, the aptamer is displaced from one set of nanocarriers, leading to a reduction in translocation velocity of that nanocarrier alone (Figure 5b). The Multiplexed assay data in comparable to the single assay results from figures 3 and 4 shown here for 
comparison. Control target incubations demonstrate that this effect is specific to the aptamer and its target. When the control target, Irinotecan, was introduced to the multiplexed assay, there was no change seen in the velocity of nanocarrier, relative to the internal standard, showing that multiple target detection was possible.

b)
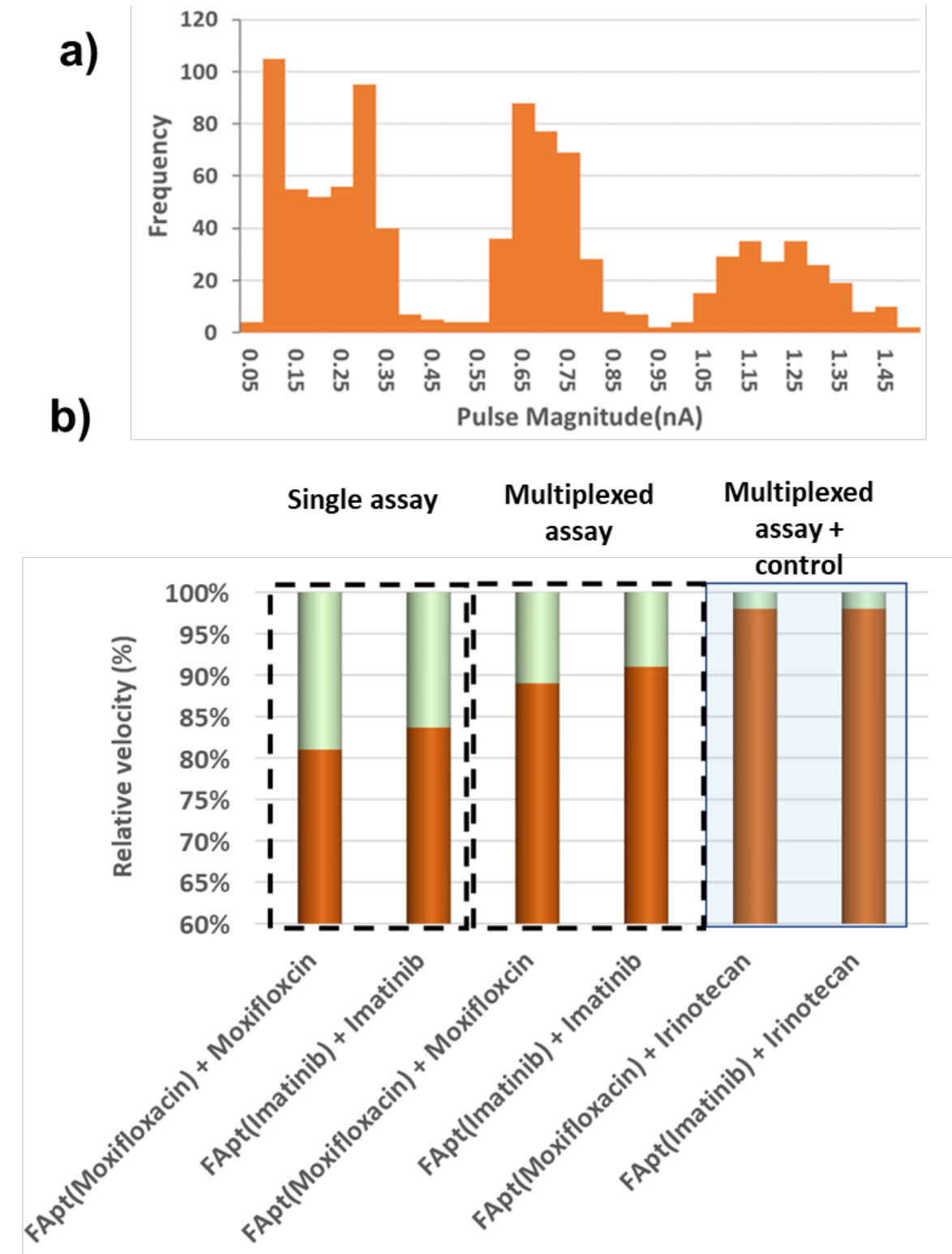

Figure 5:a) Size distributions $(\mathrm{nm})$ of the multiplexed sample with polystyrene nanocarriers sizes of 150,300 and $350 \mathrm{~nm}$ with $+0.6 \mathrm{~V}$ voltage and stretch of $48 \mathrm{~mm}$. b) Relative velocity of the different sized nanocarriers relative to the internal standard of the $350 \mathrm{~nm}$ nanocarrier.

\section{Conclusion}

Here we present a simple strategy for incorporating aptamers into RPS, whereby the signal does not depend upon the aptamer structure. The assay is carried out by immobilising an aptamer onto a nanocarrier via a ssDNA anchor sequence. In the presence of the analyte the aptamer is released from the nanocarrier surface, leaving behind a ssDNA coated nanocarrier. The change from 'aptamer coated' to 'anchor 
coated' nanocarriers is monitored via RPS. The change in signal is specific and quantitative. We use this method to detect three small molecules, of Moxifloxacin, Imatinib and Irinotecan. We also demonstrate the use of multiple nanocarriers to detect two small molecules simultaneously in a multiplexed sample.

\section{References}

1. Ellington, A. D. \& Szostak, J. W. In vitro selection of RNA molecules that bind specific ligands. Nature 346, 818-822 (1990).

2. Tuerk, C. \& Gold, L. Systematic evolution of ligands by exponential enrichment: RNA ligands to bacteriophage T4 DNA polymerase. Science (80-. ). 249, 505-510 (1990).

3. Knight, C. G. C. G. et al. Array-based evolution of DNA aptamers allows modelling of an explicit sequence-fitness landscape. Nucleic Acids Res. 37, e6 (2009).

4. Platt, M. et al. Aptamer evolution for array-based diagnostics. Anal. Biochem. 390, 203-205 (2009).

5. Cox, J. C., Rudolph, P. \& Ellington, A. D. Automated RNA selection. Biotechnol Prog 14, 845-850 (1998).

6. Bunka, D. H. J. \& Stockley, P. G. Aptamers come of age - at last. Nat Rev Micro 4, 588-596 (2006).

7. Keefe, A. D., Pai, S. \& Ellington, A. Aptamers as therapeutics. Nat Rev Drug Discov 9, 537-550 (2010).

8. Cho, E. J., Lee, J.-W. \& Ellington, A. D. Applications of aptamers as sensors. Annu. Rev. Anal. Chem. (Palo Alto. Calif). 2, 241-64 (2009).

9. Rotem, D., Jayasinghe, L., Salichou, M. \& Bayley, H. Protein detection by nanopores equipped with aptamers. J. Am. Chem. Soc. 134, 2781-7 (2012).

10. Ali, M., Nasir, S. \& Ensinger, W. Bioconjugation-induced ionic current rectification in aptamer-modified single cylindrical nanopores. Chem. Commun. 51, 3454-3457 (2015).

11. Platt, M., Willmott, G. R. G. R. \& Lee, G. U. G. U. Resistive Pulse Sensing of AnalyteInduced Multicomponent Rod Aggregation Using Tunable Pores. Small 8, 2436-2444 (2012).

12. Alsager, O. a, Kumar, S., Willmott, G. R., McNatty, K. P. \& Hodgkiss, J. M. Small molecule detection in solution via the size contraction response of aptamer functionalized nanoparticles. Biosens. Bioelectron. 57, 262-8 (2014).

13. Billinge, E. R., Broom, M. \& Platt, M. Monitoring aptamer-protein interactions using tunable resistive pulse sensing. Anal. Chem. 86, 1030-1037 (2014).

14. Heaton, I. \& Platt, M. Peptide Nanocarriers for Detection of Heavy Metal lons Using Resistive Pulse Sensing. Anal. Chem. 91, 11291-11296 (2019).

15. Billinge, E. R. \& Platt, M. Multiplexed, label-free detection of biomarkers using aptamers and Tunable Resistive Pulse Sensing (AptaTRPS). Biosens. Bioelectron. 68, 741-748 (2015).

16. Blundell, E. L. C. J., Mayne, L. J., Lickorish, M., Christie, S. D. R. \& Platt, M. Protein detection using tunable pores: resistive pulses and current rectification. Faraday Discuss. 193, 487-505 (2016).

17. Mayne, L., Lin, C.-Y. Y., Christie, S. D. R. R., Siwy, Z. S. \& Platt, M. The Design and Characterization of Multifunctional Aptamer Nanopore Sensors. ACS Nano 12, 48444852 (2018).

18. Oh, S. S., Plakos, K., Lou, X., Xiao, Y. \& Soh, H. T. In vitro selection of structureswitching, self-reporting aptamers. Proc. Natl. Acad. Sci. 107, 14053-14058 (2010).

19. Stoltenburg, R., Nikolaus, N. \& Strehlitz, B. Capture-SELEX: Selection of DNA Aptamers for Aminoglycoside Antibiotics. J. Anal. Methods Chem. 2012, 1-14 (2012).

20. Blundell, E. L. C. J., Vogel, R. \& Platt, M. Particle-by-Particle Charge Analysis of DNA-Modified Nanoparticles Using Tunable Resistive Pulse Sensing. Langmuir 32, 
1082-1090 (2016).

21. Kozak, D. et al. Simultaneous size and $\zeta$-potential measurements of individual nanoparticles in dispersion using size-tunable pore sensors. ACS Nano 6, 6990-6997 (2012).

22. Blundell, E. L. C. J., Vogel, R. \& Platt, M. Particle-by-Particle Charge Analysis of DNA-Modified Nanoparticles Using Tunable Resistive Pulse Sensing. Langmuir 32, 1082-1090 (2016).

23. Healey, M. J. M. J., Rowe, W., Siati, S., Sivakumaran, M. \& Platt, M. Rapid Assessment of Site Specific DNA Methylation through Resistive Pulse Sensing. ACS Sensors 3, 655-660 (2018). 